\title{
A new class of nitrobenzoic acid-based AIE photosensitizers for highly efficient photodynamic antibacterial therapy
}

\author{
Huanhuan Wang ${ }^{1,2}$, Xiaohong Pan ${ }^{1 *}$, Yaqi Wang ${ }^{1}$, Wenzhen Liu ${ }^{1}$, Tao Dai ${ }^{1,2}$, Binbin Yuan $^{1}$, \\ Xueyuan Chen ${ }^{1,2}$ and Zhuo Chen ${ }^{1,2^{*}}$
}

\begin{abstract}
Photodynamic therapy (PDT) has been drawing more and more attention in the antibacterial field. Traditional photosensitizers (PSs) tend to aggregate in aqueous media, which reduces the generation of reactive oxygen species (ROS) and seriously affects the photodynamic efficacy. Many efforts have been made to prevent aggregation of traditional PSs. By contrast, aggregation-induced emission PSs (AIE-PSs) take advantage of aggregation to boost ROS generation and fluorescence intensity. However, the efficacies of the reported antibacterial AIE-PSs are poor. Herein, we report a new class of highly effective antibacterial AIE-PSs based on nitrobenzoic acid structure. TTVBA, a negatively charged AIE-PS, can not only selectively kill spherical bacteria (Staphylococcus aureus (S. aureus)) rather than rod-shaped bacteria (Escherichia coli (E. coli)), but also be easily extended to several AIE-PSs (TTVBP1-3) with positive charges and broad-spectrum antibacterial activity. We demonstrate that TTVBP2 can kill $3.0 \log _{10}$ of $S$. aureus at very low concentration $\left(125 \mathrm{nmol} \mathrm{L}^{-1}\right)$, TTVBP3 can kill $4.7 \log _{10}$ of Staphylococcus epidermidis (S. epidermidis) at a concentration of $1 \mu \mathrm{mol} \mathrm{L}^{-1}$ and $3.8 \log _{10}$ of E. coli at $5 \mu \mathrm{mol} \mathrm{L} \mathrm{L}^{-1}$, thus enabling them among the most effective antibacterial AIE-PSs reported so far. Meanwhile, these AIE-PSs exhibit excellent wash-free imaging ability for bacteria by simple mixing with bacteria. We thus envision that TTVBA, a nitrobenzoic acid-based extendable AIE-PS, provides a new route for the design of AIE-PSs in antibacterial treatment.
\end{abstract}

Keywords: photodynamic antibacterial therapy, photosensitizer, aggregation-induced emission, reactive oxygen species, imaging

\section{INTRODUCTION}

With the long-term overuse and abuse of antibiotics, the number of drug-resistant bacteria is increasing, and thus bacterial infection has been a serious threat to human health $[1,2]$. Photodynamic therapy (PDT), which utilizes photosensitizers (PSs), light and oxygen to generate toxic reactive oxygen species (ROS) to destroy the external and internal structures of bacteria, is considered not susceptible to drug resistance and to be an effective way to solve this serious medical problem [3-8].

Traditional PSs, such as porphyrin, phthalocyanine and boron-dipyrromethene, are prone to forming aggregates in aqueous media $[4,9]$. The strong $\pi-\pi$ stacking in the aggregate quenches the fluorescence, decreases the ROS generation, reduces the signal-to-noise ratio of bacterial imaging, and decreases the sterilization effect [10-14]. By contrast, aggregation-induced emission PSs (AIE-PSs) emit weak light in molecular species, but shine up upon aggregation due to the restriction of intramolecular motions which suppresses nonradiative decay and results in high ROS generation [11,12,15-17]. Since the first discovery of AIE phenomenon in 2001 [18], AIE-PSs have been successfully applied in the cell $[12,19-26]$ and bacteria imaging and ablation [4,27-38]. In addition to the membrane-anchoring AIE-PS reported in 2019 that killed $99.9 \%\left(3.0 \log _{10}\right)$ of Staphylococcus aureus (S. aureus) at a concentration of $2 \mu \mathrm{mol} \mathrm{L}^{-1}$ and $99.9 \%$ of $E$. coli at $5 \mu \mathrm{mol} \mathrm{L}^{-1}$ [39], most reported antibacterial rates of AIEPSs did not exceed 99\% $\left(2.0 \log _{10}\right)$. Therefore, more effective antibacterial AIE-PSs, especially AIE-PS backbones that can be easily added with different anchoring

\footnotetext{
${ }^{1}$ State Key Laboratory of Structural Chemistry, CAS Key Laboratory of Design and Assembly of Functional Nanostructures, Fujian Institute of Research on the Structure of Matter, Chinese Academy of Sciences, Fuzhou 350002, China

${ }^{2}$ University of Chinese Academy of Sciences, Beijing 100049, China

* Corresponding authors (emails: panxiaohong@fjirsm.ac.cn (Pan X); zchen@fjirsm.ac.cn (Chen Z))
} 
groups for bacteria are still much needed.

In addition, due to the negative charges on the surface of bacteria, most PSs are designed to have positive charges, which bind to all bacteria through electrostatic interaction without selectivity $[4,40,41]$. To reduce the impact of off-target on microbial community (e.g., intestinal microflora), more and more attention has been paid to selective killing methods of harmful pathogens [42-45]. Recently, it has been reported that negatively charged nanospheres, driven by entropy gain, could adsorb selectively onto spherical bacteria, but not onto rodshaped bacteria [3]. Verteporfin and chlorin e6 as the traditional PSs were preloaded into nano-micelles to prevent aggregation and quenching of ROS generation. The resulting photodynamic nanospheres with negative charges killed more than $99 \%$ of spherical bacteria but less than $1 \%$ of rod-shaped bacteria [3]. Rather than quenching ROS generation for traditional PSs [46], aggregation boosts ROS generation for AIE-PSs [47]. Therefore, AIE-PSs are promising candidates to form photodynamic nanospheres by simple aggregation without further decoration. Although some AIE luminogens with negative charges have been reported [48-50], few AIE-PSs with negative charges have been studied.

In this contribution, we designed and synthesized TTVBA, a novel extendable AIE-PS (Scheme 1). The nitrobenzoic acid fragment endows TTVBA with a negative charge in physiological environment. TTVBA as the backbone was extended to TTVBP and TTVBP1-3 by coupling with piperazine and alkyl halides. TTVBP1 has a positive charge near the backbone, while TTVBP2 has a positive charge at the end of the chain (away from the backbone). TTVBP3 is a hybrid of TTVBP1 and TTVBP2 with two positive charges. Their structurefunction relationships toward narrow- and broad-spectrum bacterial ablation and imaging were evaluated. This study demonstrates that TTVBA is a promising AIE-PS backbone, which can be extended into AIE-PSs with high antibacterial efficiency.

\section{EXPERIMENTAL SECTION}

\section{Chemicals}

Singlet oxygen sensor green (SOSG) were obtained from Shanghai Aladdin Bio-Chem Technology Co., Ltd. Phosphate buffer saline (PBS, pH 7.4) used throughout the work was purchased from Dalian Meilun Biotechnology Co., Ltd. Silica gel, alumina and basic alumina were purchased from Shanghai Aladdin Bio-Chem Technology Co., Ltd. All solvents and materials were used as received without further purification. TTVBA, TTVBP and TTVBP1-3 were pre-dissolved in dimethyl sulfoxide (DMSO, $1 \mathrm{mmol} \mathrm{L}^{-1}$ ) throughout the experiments.

\section{Instruments}

${ }^{1} \mathrm{H}$ and ${ }^{13} \mathrm{C}$ NMR spectra were obtained on a Bruker BioSpin AVANCE III $400 \mathrm{MHz}$ spectrometer or a JEOL EZC400S $400 \mathrm{MHz}$ spectrometer. High-resolution mass spectra (HRMS) were recorded on a Bruker Impact II Mass Spectrometer. Absorption and fluorescence spectra were measured on a BioTek Synergy ${ }^{\mathrm{TM}} 4$ Multi-Mode Microplate Reader. The binding kinetics of AIE-PSs with bacteria and the uptake of AIE-PSs by bacteria were detected on a CytoFLEX flow cytometer (Beckman Coulter Inc., CA, USA). The images of bacteria were observed by an Olympus FluoView ${ }^{\mathrm{TM}}$ FV1000 confocal laser scanning microscope (CLSM). The particle sizes and zeta potentials were measured by a dynamic light scattering (DLS) instrument (Zetasizer 3000, Malvern Instruments, Ltd.). The morphologies were characterized by using a fieldemission scanning electron microscope (SEM, JEOLJSM-6700F) at $10 \mathrm{kV}$.

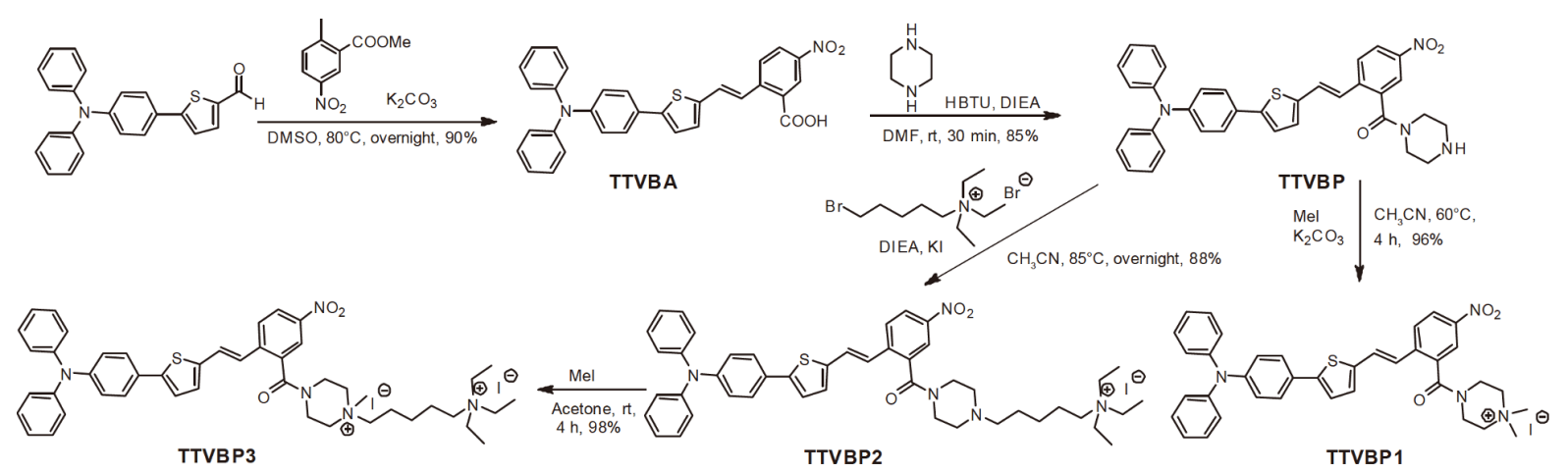

Scheme 1 Synthesis of TTVBA and its derivatives. HBTU: 2-(1H-benzotriazole-1-yl)-1,1,3,3-tetramethyluronium hexafluorophosphate; DIEA: N,Ndiisopropylethylamine; DMF: $N, N$-dimethylformamide; MeI: iodomethane; rt: room temperature. 


\section{Bacterial strains and cultivation conditions}

Gram-positive bacteria S. aureus (ATCC 6538), Staphylococcus epidermidis (S. epidermidis, ATCC 12228), Enterococcus faecalis (E. faecalis, ATCC 29212) and Gramnegative bacterium Escherichia coli (E. coli, ATCC 25922) were purchased from Beijing Zhongyuan Ltd. (China). They were grown in Luria-Bertani (LB) broth media (1\% tryptone, $0.5 \%$ yeast extract, $1 \% \mathrm{NaCl}, \mathrm{pH} 7.0$ ) at $37^{\circ} \mathrm{C}$ with shaking at $220 \mathrm{r} \mathrm{min}^{-1}$. The number of bacteria was estimated by measuring the optical density at $600 \mathrm{~nm}$ to obtain $10^{8} \mathrm{CFU} \mathrm{mL}^{-1}$ (colony forming unit, $\mathrm{CFU}$ ).

\section{Cell line and culture}

The human normal liver cell line L-O2 was purchased from Shanghai Institute of Cell Biology, Chinese Academy of Sciences (China), and maintained in Dulbecco's Modified Eagle's Medium (DMEM) with 10\% fetal bovine serum (Gibco, Invitrogen, Grand Island, NY, USA), $100 \mu \mathrm{g} \mathrm{m}^{-1}$ penicillin and $100 \mu \mathrm{g} \mathrm{mL}^{-1}$ streptomycin (Gibco, Invitrogen, Grand Island, NY, USA) at $37^{\circ} \mathrm{C}$ with $5 \% \mathrm{CO}_{2}$.

\section{Synthesis of TTVBA, TTVBP and TTVBP1-3}

The synthetic methods and the characterizations of TTVBA, TTVBP and TTVBP1-3 $\left({ }^{1} \mathrm{H}\right.$ NMR, ${ }^{13} \mathrm{C}$ NMR and mass spectra) are detailed in the Supplementary information (Figs S1-S10).

\section{Singlet oxygen generation}

The generation of singlet oxygen in the photodynamic therapy of AIE-PSs under white-light irradiation was studied by using the molecular probe SOSG. In the presence of singlet oxygen, SOSG can react with singlet oxygen to produce SOSG endoperoxides (SOSG-EPs) that emit strong green fluorescence $\left(\lambda_{\mathrm{ex}}=488 \mathrm{~nm}, \lambda_{\mathrm{em}}=\right.$ $520 \mathrm{~nm})$. Typically, PBS $(200 \mu \mathrm{L})$ containing SOSG $\left(100 \mu \mathrm{mol} \mathrm{L}{ }^{-1}\right)$ and AIE-PS $\left(10 \mu \mathrm{mol} \mathrm{L}^{-1}\right)$ was irradiated for $20 \mathrm{~min}$, and then the fluorescence intensity at $520 \mathrm{~nm}$ was recorded every $2 \mathrm{~min}$. The solution with only SOSG was used as a control group.

\section{Photodynamic antibacterial activity}

The antibacterial activities of AIE-PSs were evaluated by the method of colony counting on an agar plate. The bacteria in PBS $\left(10^{7} \mathrm{CFU} \mathrm{mL} \mathrm{m}^{-1}, 1 \mathrm{~mL}\right)$ mixed with AIEPSs were incubated in multiple-well plates for $5 \mathrm{~min}$ in the dark at $37^{\circ} \mathrm{C}$. After 40 min of white-light irradiation $\left(400-800 \mathrm{~nm}, 20 \mathrm{~mW} \mathrm{~cm}^{-2}\right)$, aliquots $(100 \mu \mathrm{L})$ from each well were taken and serially diluted 10-fold in PBS. One hundred microlitre of each dilution was then dispersed on the LB solid agar plate to determine the CFU. One group without receiving light served as the control. The colonies were counted after incubation at $37^{\circ} \mathrm{C}$ for 16-24 h. Each experiment was performed independently at least three times.

\section{Binding kinetics of AIE-PSs with bacteria}

The binding kinetics of AIE-PSs with bacteria was monitored via a CytoFLEX flow cytometer at a fluorescent channel (PC5.5-A, $\lambda_{\mathrm{ex}}=488 \mathrm{~nm}, \quad \lambda_{\mathrm{em}}=$ $665-715 \mathrm{~nm})$. The cultured bacteria $\left(\mathrm{OD}_{600}=0.6\right)$ were centrifuged and re-suspended in PBS $(900 \mu \mathrm{L})$ by 10 -fold dilution. After being mixed with PBS solution of AIE-PS $\left(100 \mu \mathrm{mol} \mathrm{L}^{-1}, 100 \mu \mathrm{L}\right)$, the fluorescence intensity was monitored for continuous $7 \mathrm{~min}$. One group without adding AIE PS served as a control.

\section{Uptake of AIE-PSs by bacteria}

The uptake of AIE-PSs by bacteria was investigated via a CytoFLEX flow cytometer at a fluorescent channel (PC5.5-A, $\lambda_{\text {ex }}=488 \mathrm{~nm}, \lambda_{\text {em }}=665-715 \mathrm{~nm}$ ). In brief, bacteria $\left(\mathrm{OD}_{600}=0.6\right)$ were first incubated with AIE-PS $\left(10 \mu \mathrm{mol} \mathrm{L}{ }^{-1}\right)$ in PBS at $37^{\circ} \mathrm{C}$ for $5 \mathrm{~min}$ in the dark. After centrifugation at $5000 \mathrm{r} \mathrm{min}^{-1}$ for $3 \mathrm{~min}$, the precipitate was resuspended in PBS by 10 -fold dilution. The fluorescence intensity was measured and analyzed on a $\mathrm{Cy}$ toFLEX flow cytometer. One group without adding AIE PSs served as a control. Each experiment was performed independently at least three times.

\section{Zeta potential $(\zeta)$ measurements}

The zeta potential measurements were conducted for bacteria incubated with AIE-PSs using the same device for DLS measurements. In brief, bacteria $\left(\mathrm{OD}_{600}=0.6\right)$ were incubated with AIE-PS $\left(10 \mu \mathrm{mol} \mathrm{L}^{-1}\right)$ in PBS for $5 \mathrm{~min}$ in the dark at $37^{\circ} \mathrm{C}$. Following that, the bacteria were harvested after centrifugation at $5000 \mathrm{r} \mathrm{min}^{-1}$ for $3 \mathrm{~min}$ and being washed twice with water. The bacteria were then resuspended in water by 10 -fold dilution for zeta potential measurements. The bacterial samples that were not incubated with AIE-PS were used as negative controls.

\section{Bacterial imaging}

Bacteria $\left(\mathrm{OD}_{600}=0.6\right)$ were harvested by centrifugation at $5000 \mathrm{r} \mathrm{min}^{-1}$ for $3 \mathrm{~min}$ and resuspended in PBS $(990 \mu \mathrm{L})$ to receive a concentration of $10^{9} \mathrm{CFU} \mathrm{mL}^{-1}$. After being mixed with AIE-PS $\left(1 \mathrm{mmol} \mathrm{L}^{-1}\right.$ in DMSO, $\left.10 \mu \mathrm{L}\right)$ and incubated for $20 \mathrm{~min}$ in the dark at $37^{\circ} \mathrm{C}$, the bacteria were re-dispersed at room temperature. The suspension 
$(15 \mu \mathrm{L})$ was then transferred onto a glass slide and covered with a glass coverslip $(18 \mathrm{~mm} \times 18 \mathrm{~mm})$. Images were taken on an Olympus FluoView ${ }^{\mathrm{TM}}$ FV1000 CLSM $\left(\lambda_{\mathrm{ex}}=488 \mathrm{~nm}, \lambda_{\mathrm{em}}=570-1000 \mathrm{~nm}\right)$.

\section{Photostability test of AIE-PSs}

The photostability of AIE-PSs was evaluated by measuring the fluorescence intensities of AIE-PSs on S. aureus by continuous irradiation $\left(\lambda_{\mathrm{ex}}=488 \mathrm{~nm}\right)$ and sequential scanning (every $5 \mathrm{~s}, 120$ times) on an Olympus FluoView ${ }^{\mathrm{TM}}$ FV1000 CLSM.

\section{Biocompatibility tests of AIE-PSs}

The biocompatibility of AIE-PSs was evaluated by measuring the phototoxicity and dark toxicity of AIE-PSs to L-O2 cells using MTT (3-(4,5-dimethylthiazol-2-yl)-2,5diphenyltetrazolium bromide) assay. In brief, aliquots $(100 \mu \mathrm{L})$ of L-O2 cells $\left(10^{4}\right.$ cells) were cultured at $37^{\circ} \mathrm{C}$ in a 96-well plate, allowed to attach overnight, and then left untreated or treated with growth medium containing AIE-PSs with different concentrations $(6.25,12.5,25,50$, $100 \mu \mathrm{mol} \mathrm{L}^{-1}$ ) for $2 \mathrm{~h}$. The cultures were illuminated with white light $\left(20 \mathrm{~mW} \mathrm{~cm}^{-2}\right)$ for $40 \mathrm{~min}$, followed by incubation for $24 \mathrm{~h}$. Freshly prepared MTT solution $\left(5 \mathrm{mg} \mathrm{mL}^{-1}, 10 \mu \mathrm{L}\right)$ was then added into each well, followed by incubation for another $4 \mathrm{~h}$. After removing the MTT containing medium, $150 \mu \mathrm{L}$ of DMSO was added to dissolve the Formosan crystals. The optical density of solution was measured by enzyme-linked immunosorbent assay (ELISA) at $490 \mathrm{~nm}$. The value of cell viability was determined according to the following formula: cell viability $(\%)=$ (mean absorbance of the treatment group/ mean absorbance of the control group) $\times 100 \%$. The dark toxicity of AIE-PSs to L-O2 cells in the absence of light was measured in parallel.

\section{Statistical analysis}

All data represent group means and standard errors (SEs) of the mean. The data of antibacterial activity were analyzed by two-way analyses of variance (ANOVA). The data of binding capacity of AIE-PSs by bacteria was analyzed by one-way ANOVA. Individual group means were compared by the Newman-Keuls multiple range test.

\section{RESULTS AND DISCUSSION}

\section{Molecular design and synthesis}

As shown in Scheme 1, Knoevenagel condensation of triphenylamine-thiophene and methyl 2-methyl-5- nitrobenzoate afforded TTVBA as a red precipitate in $90 \%$ yield without further purification. The following amidation of TTVBA with piperazine gave TTVBP in a yield of $85 \%$. Alkylation of TTVBP with methyl iodide and 5-bromo- $N, N, N$-triethylpentan-1-aminium bromide provided TTVBP1, TTVBP2 and TTVBP3, respectively in high yield. TTVBA as the backbone structure consists of a triphenylamine moiety (electron donor and spacer to prevent $\pi-\pi$ stacking in the aggregate), a thiophene fragment (electron donor and $\pi$-bridge), a carbon-carbon double bond ( $\pi$-bridge) and a nitrobenzoic acid unit (electron acceptor). This structure possesses extended $\pi$ conjugation and strong donor-acceptor effect which will cause the separation of the highest occupied molecular orbital (HOMO) and the lowest unoccupied molecular orbital (LUMO), decrease the singlet-triplet energy gap and thus increase the ROS generation capacity $[23,45,51]$. The carboxylic acid moiety, working as an electron withdrawing group, will not only bring TTVBA a negative charge in physiological environment, but also make TTVBA a versatile backbone to conjugate with amines, alcohols, amino acids, peptides and proteins to achieve different functionalities.

\section{Physical properties}

Considering TTVBP is also the backbone of TTVBP1-3, we chose TTVBA and TTVBP as the representatives of new AIE-PSs to study their physical properties. As depicted in Fig. 1a, the absorptions of TTVBA and TTVBP in DMSO are broad from 400 to $550 \mathrm{~nm}$ in the visiblelight range, and the peaks locate at 447 and $457 \mathrm{~nm}$, respectively. The AIE features of TTVBA and TTVBP were demonstrated by testing their emission in a mixed solvent (DMSO/toluene) with different toluene fractions (Fig. 1b-d). TTVBA and TTVBP behaved similarly in these tests. No emission was observed in DMSO (well dissolved), but the emission enhanced gradually with the increase of toluene fraction up to $90 \%$. A dramatic enhancement of emission was observed when the toluene fraction was increased to $95 \%$ and $99 \%$, and their maximum peaks (around $640 \mathrm{~nm}$ ) were 360 times higher than those in pure DMSO solution. This emission enhancement is mostly due to the restriction of intramolecular motions when AIE-PSs aggregate in toluene (poor solvent). Because of the donor-acceptor structure in TTVBA and TTVBP, the emission peaks blue-shift gradually from DMSO (polar solvent) to toluene (nonpolar solvent), which is a typical twisted intramolecular charge transfer effect $[52,53]$. The emission and absorption characteristics of TTVBP1-3 (Fig. S11) were similar to that of 

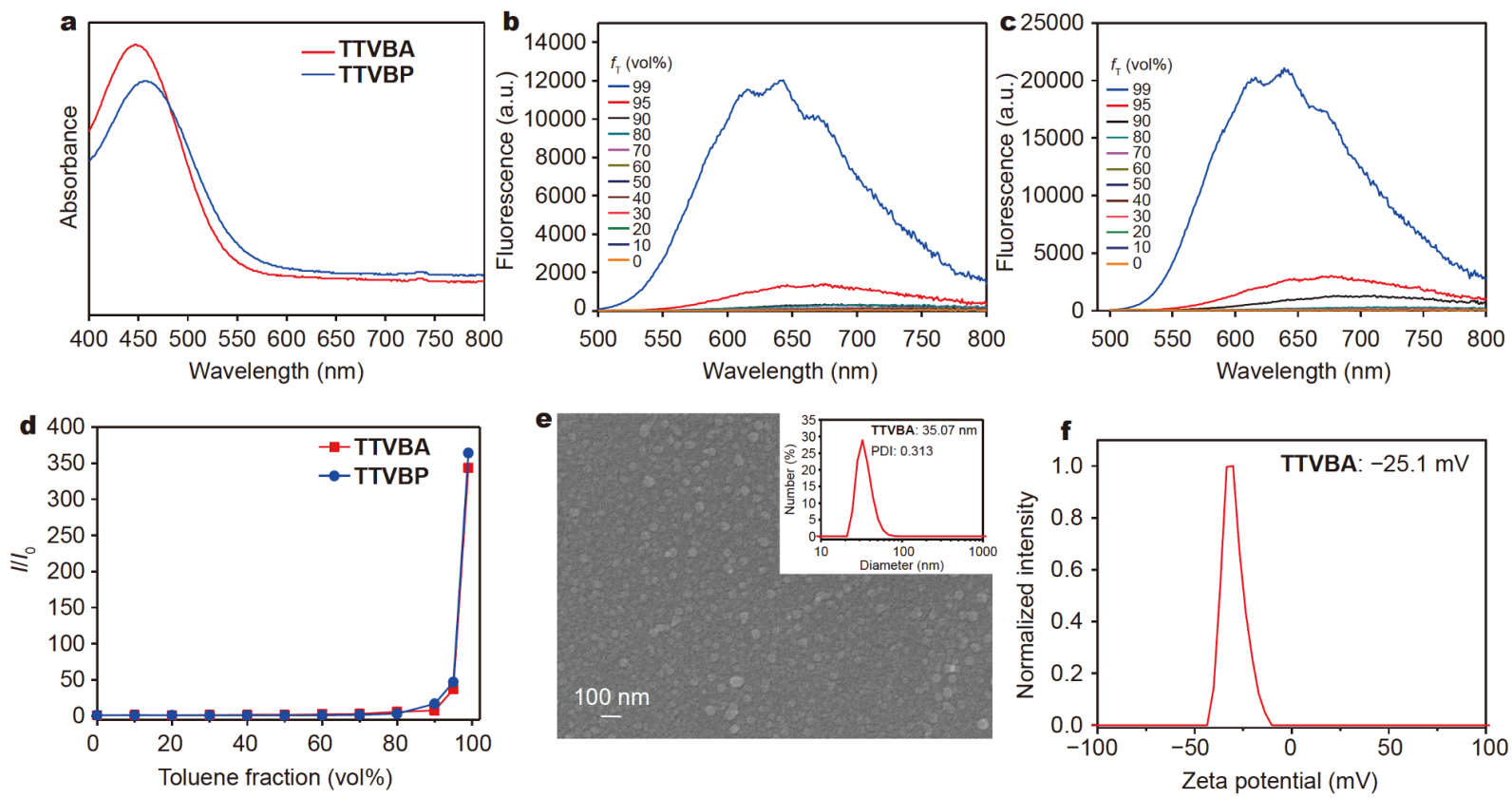

Figure 1 Physical properties of AIE-PSs TTVBA and TTVBP. (a) Absorption spectra of TTVBA and TTVBP in DMSO. Photoluminescence spectra of (b) TTVBA $\left(10^{-5} \mathrm{~mol} \mathrm{~L}^{-1}\right)$ and (c) TTVBP $\left(10^{-5} \mathrm{~mol} \mathrm{~L}^{-1}\right)$ in mixed solvents with different toluene fractions (DMSO/toluene). (d) Plots of relative emission intensity of TTVBA and TTVBP versus toluene fraction. $I_{0}$ and $I$ are the peak values of photoluminescence intensities in DMSO and in mixed solvent (DMSO/toluene), respectively. (e) SEM image of TTVBA $\left(10^{-6} \mathrm{~mol} \mathrm{~L}^{-1}\right)$ in $1 \% \mathrm{DMSO} /$ water and hydrodynamic size distribution of TTVBA $\left(10^{-6} \mathrm{~mol} \mathrm{~L}^{-1}\right)$ in $1 \%$ DMSO/PBS (inset). (f) Zeta potential of TTVBA $\left(10^{-6} \mathrm{~mol} \mathrm{~L}^{-1}\right)$ in $1 \% \mathrm{DMSO} /$ water.

TTVBP. The different intensities among them may be due to the difference of charge number and position, leading to their different solubilities and aggregation intensities in the solvent. DLS analysis (Fig. 1e inset and 1f) demonstrated that TTVBA formed negatively charged nanospheres in physiological environment $(\sim 35 \mathrm{~nm}$ diameter, $-25 \mathrm{mV}$ zeta potentials). The image obtained from SEM (Fig. 1e) showed that the nanospheres have an average diameter of $\sim 39 \mathrm{~nm}$.

\section{Theoretical calculation and singlet oxygen generation ability}

In order to further evaluate the structural design of our AIE-PSs, density functional theory (DFT) calculations of TTVBA and TTVBP were performed (Fig. 2a and b). The calculated HOMO-LUMO energy gaps of TTVBA and TTVBP are 2.37 and $2.49 \mathrm{eV}$, respectively. Their LUMOs are mainly delocalized at nitrobenzene and carbon-carbon double bond units, while triphenylamine and thiophen groups dominate the HOMOs. The strong donoracceptor effect separates their HOMO and LUMO. Because of this separation, we were expecting their high ROS generation ability [54].

Among ROS, including superoxide and hydroxyl radicals, singlet oxygen is the primary cytotoxic agent to destruct biological tissues and cells in PDT because of its strong diffusibility and high oxidative efficiency [55-57]. Therefore, the singlet oxygen generation abilities of TTVBA and TTVBP were evaluated in this study using SOSG, a specific singlet oxygen probe. Commercial PSs Rose Bengal (RB) and chlorin e6 (Ce6) were used as references in current study. SOSG alone was nonfluorescent, while its fluorescence gradually enhanced when exposed to white light in the presence of above PSs, indicating the generation of singlet oxygen (Fig. 2c). The fluorescence intensity of SOSG treated with TTVBA or TTVBP was significantly higher than that of SOSG treated with RB or Ce6. Furthermore, the fluorescence intensity of SOSG treated with TTVBA was stronger than that of SOSG treated with TTVBP, demonstrating that TTVBA has stronger singlet oxygen generation ability than TTVBP.

\section{Photodynamic antibacterial study}

As both TTVBA and TTVBP have strong singlet oxygen generation abilities, it motivated us to explore the photodynamic antibacterial performances of TTVBA and TTVBP1-3 against Gram-positive spherical S. aureus and Gram-negative rod-shaped $E$. coli under white-light irradiation $\left(400-800 \mathrm{~nm}, 20 \mathrm{~mW} \mathrm{~cm}{ }^{-2}\right)$ for $40 \mathrm{~min}$. The 

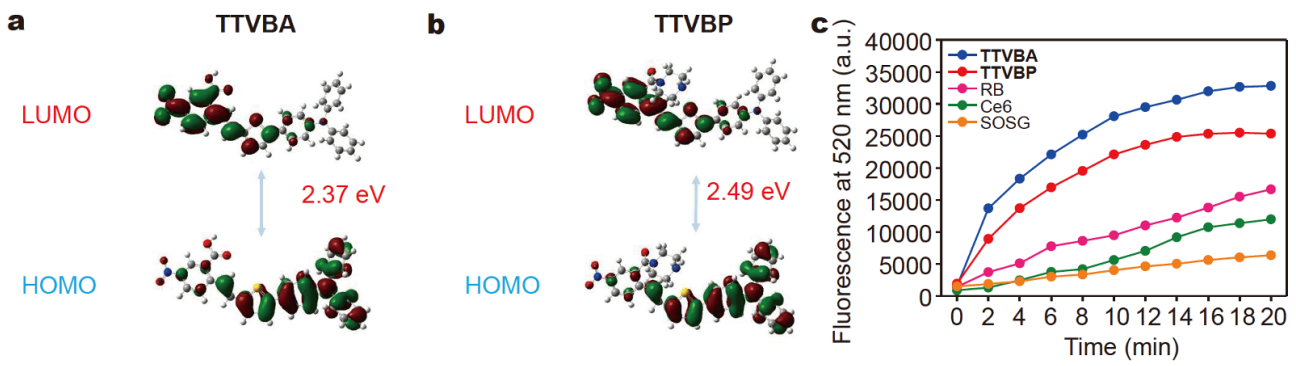

Figure 2 Theoretical calculation and singlet oxygen generation abilities of AIE PSs TTVBA and TTVBP. Theoretical calculations for frontier molecular orbitals of (a) TTVBA and (b) TTVBP were performed by using the density functional theory (DFT) at B3LYP/6-31G* level via the Gaussian 09 program. (c) Singlet oxygen produced by TTVBA and TTVBP.
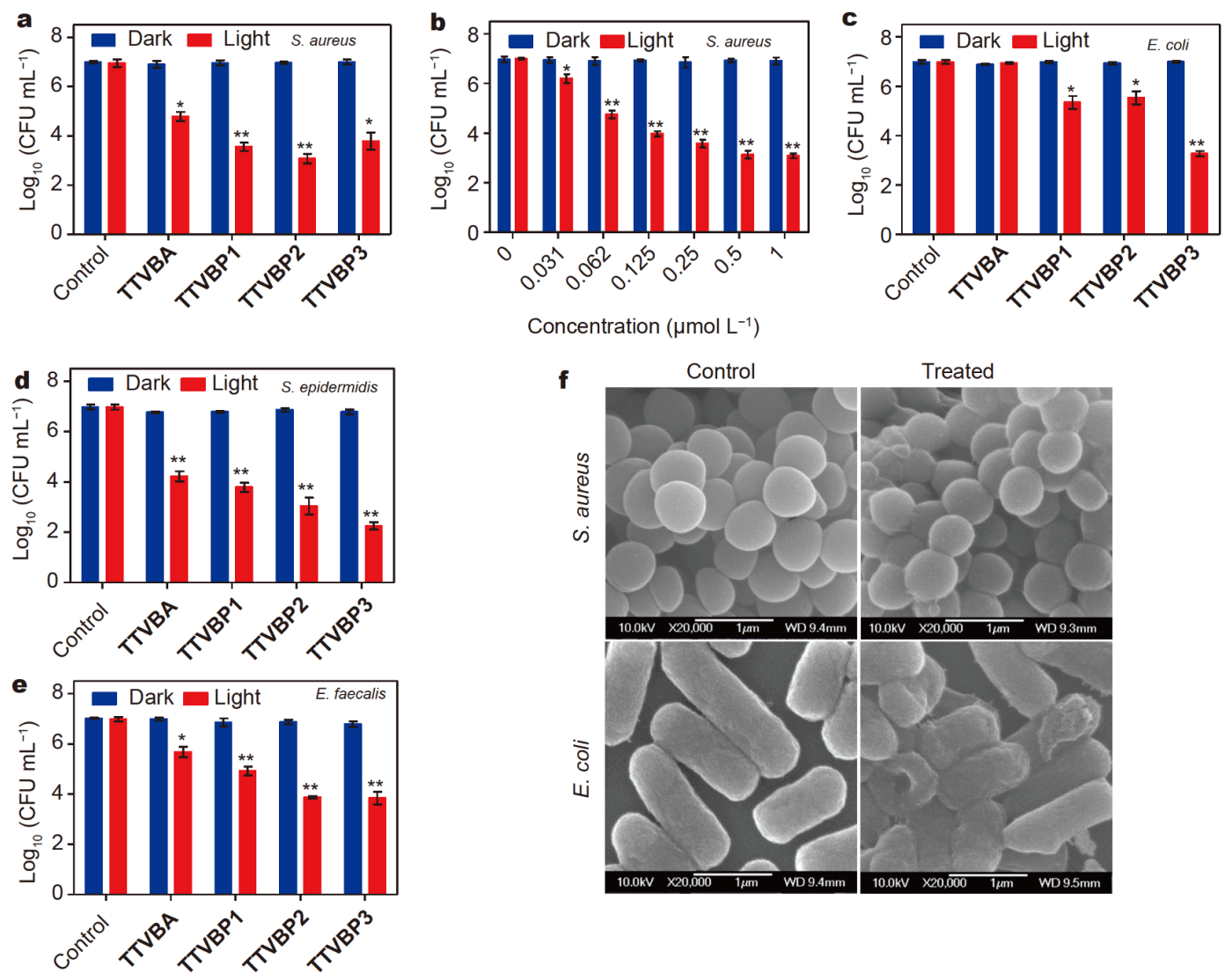

Figure 3 Photodynamic antibacterial activities of AIE-PSs. (a) Photodynamic antibacterial activities of AIE-PSs $\left(10^{-6} \mathrm{~mol} \mathrm{~L}^{-1}\right)$ against $S$. aureus under white-light irradiation $\left(20 \mathrm{~mW} \mathrm{~cm}^{-2}, 40 \mathrm{~min}\right)$. (b) Dose-response relationship of TTVBP2 against $S$. aureus under white-light irradiation. Photodynamic antibacterial activities of AIE-PSs against (c) E. coli $\left(5 \times 10^{-6} \mathrm{~mol} \mathrm{~L}^{-1}\right)$, (d) S. epidermidis $\left(10^{-6} \mathrm{~mol} \mathrm{~L}^{-1}\right)$ and (e) E. faecalis $\left(10^{-6} \mathrm{~mol} \mathrm{~L}^{-1}\right)$ under white-light irradiation. (f) SEM images of $S$. aureus and E. coli before and after treatment with TTVBP2 under white-light irradiation. Data are given as mean $\pm \mathrm{SE}(n=3){ }^{*}$ and ${ }^{*}$ indicate significant differences $(P<0.05$ and $P<0.01$, respectively) from the corresponding control group.

antibacterial effect was demonstrated by traditional agar plate CFU counting method. TTVBP was not further investigated because it exists as a neutral molecule and can neither kill nor stain $S$. aureus or E. coli in the preexperiments. As shown in Fig. 3a, TTVBP1-3 $\left(1 \mu \mathrm{mol} \mathrm{L}^{-1}\right)$ had significant scavenging effects on $S$. aureus (3.3-4.0 $\left.\log _{10}\right)$ compared with TTVBA $\left(2.2 \log _{10}=\right.$ 99.2\%). This may be due to the fact that negatively charged TTVBA nanospheres cannot bind to bacteria as strong as positively charged TTVBP1-3 which interact with bacteria by electrostatic adsorption. No dark toxicity to $S$. aureus was found in this study. Taking TTVBP2 as 
an example, the dose-response relationship of TTVBP2 against $S$. aureus was explored (Fig. 3b). TTVBP2 had a dose-dependent bactericidal activity against $S$. aureus, killing $3.0 \log _{10}(99.9 \%)$ of $S$. aureus at a concentration of $125 \mathrm{nmol} \mathrm{L}^{-1}$. To the best of our knowledge, this is the first report describing that AIE-PS has good bactericidal activity at such a low concentration $\left(125 \mathrm{nmol} \mathrm{L}^{-1}\right)$.

The photodynamic antibacterial activities of AIE-PSs $\left(5 \mu \mathrm{mol} \mathrm{L}^{-1}\right)$ against E. coli were illustrated in Fig. 3c. Among the AIE-PSs investigated, TTVBP3 had the strongest bactericidal effect on $E$. coli with a killing rate of $3.8 \log _{10}$ (the killing rate of TTVBP1 and TTVBP2 to $E$. coli was $1.7 \log _{10}$ and $1.5 \log _{10}$, respectively), making TTVBP3 one of the most effective AIE-PSs reported so far to kill E. coli. No cytotoxicity of TTVBA was found to E. coli, mainly because the negatively charged TTVBA nanospheres cannot bind to rod-shaped bacteria [3]. Similarly, no dark toxicity to $E$. coli was observed in this study. In a word, TTVBP1-3 are excellent broad-spectrum antibacterial AIE-PSs, while TTVBA is a potential narrow-spectrum antibacterial AIE-PS that can kill spherical bacteria instead of rod-shaped bacteria.

We further investigated the antibacterial effects of AIEPSs against S. epidermidis and E. faecalis. As shown in Fig. 3d and e, AIE-PSs $\left(1 \mu \mathrm{mol} \mathrm{L}{ }^{-1}\right)$ were efficient to kill $S$. epidermidis (3.1-4.7 $\left.\log _{10}\right)$ in the presence of white-light irradiation (40 $\mathrm{min}, 20 \mathrm{~mW} \mathrm{~cm}^{-2}$ ). By comparison, $E$. faecalis was more tolerant to PDT treatment due to its thick cell wall. The killing rates of TTVBA and TTVBP1 $\left(1 \mu \mathrm{mol} \mathrm{L}{ }^{-1}\right)$ were $1.3 \log _{10}$ and $2.1 \log _{10}$, respectively. It is worth mentioning that TTVBP2 and TTVBP3 could kill $3.2 \log _{10}$ of E. faecalis.

In order to gain more information about the underlying mechanisms, micrographs of $S$. aureus or E. coli treated with TTVBP2 under white-light irradiation $(40 \mathrm{~min}$, $20 \mathrm{~mW} \mathrm{~cm}^{-2}$ ) were obtained by SEM (Fig. 3f). It was observed that the outer surfaces of both $S$. aureus and $E$. coli became rough, dented and damaged (fusion of cell walls and leakage of intracellular contents) after the PDT with TTVBP2.

\section{Binding capacity and bacterial imaging}

TTVBA is the backbone of TTVBP1-3, and they differ in the functional groups (alkylated piperazine with positive charges) that help them bind to bacteria. Therefore, their different antibacterial efficiencies are mostly due to their different binding capacities with bacteria [4]. When AIEPSs aggregate or bind to cell membrane, their intramolecular motion is restricted, which suppresses the nonradiative decay and results in ROS generation and light emission [45]. Accordingly, the fluorescence intensity of AIE-PSs on bacteria reflects their binding capacities with bacteria [4]. This makes it possible to detect the binding kinetics between AIE-PSs and bacteria by tracking the fluorescence change on bacteria using a flow cytometer (Fig. 4, Figs S12-S14) [41]. The fluorescence intensity of $S$. aureus reached a maximum value (29-fold increased) within 3-min incubation with AIE-PSs, while that of E. coli hardly changed when treated with TTVBA, indicating that TTVBA was bound to $S$. aureus, but not to $E$. coli. This phenomenon is consistent with the selective killing effect of TTVBA we observed previously on $S$. aureus rather than E. coli. On the contrary, E. coli incubated with TTVBP1-3 exhibited strong fluorescence intensity in $3 \mathrm{~min}$, demonstrating the binding activity of TTVBP1-3 with E. coli. It was noticeable that the fluorescence intensity of TTVBP3 was 1.5 -fold higher than those of TTVBP1 and TTVBP2, revealing the strongest binding capacity of TTVBP3 with E. coli. These results may be due to the fact that TTVBP3 has two positive charges, which could replace the divalent cations $\left(\mathrm{Ca}^{2+}\right.$ or $\left.\mathrm{Mg}^{2+}\right)$ on lipid A that stabilize the lipopolysaccharide (LPS) structure, and interact strongly with the negatively charged LPS on the outer membrane of Gramnegative bacteria. The resulting unstable LPS coating forms "cracks" in the permeability barrier, which enable TTVBP3 to penetrate into the periplasmic space $[58,59]$. The different binding capacities of our AIE-PSs with bacteria suggested that alkylated piperazine with two positive charges could be an effective bacterial membrane-anchoring group.

To further investigate the binding site of bacteria and AIE-PSs, zeta potential of bacteria, which reflects the surface charge of bacteria, was measured in this study. The positive charges of TTVBP1-3 could be exposed on the surface of bacteria if they only bound to the surface of bacteria, making the zeta potential of bacteria more positive $[60,61]$. However, no change in zeta potential of $S$. aureus or $E$. coli was observed when treated with TTVBP1-3 (Fig. S15), indicating that TTVBP1-3 can effectively insert into the bacterial cell wall instead of just binding to the bacterial surface [45].

TTVBA, a negatively charged nanosphere, was found to bind onto spherical-like $S$. aureus rather than rodshaped E. coli, thus keeping zeta potential of E. coli unchanged. On the other hand, TTVBA lighted up S. aureus as strongly as TTVBP1-3 did in 1\% DMSO/PBS (Fig. 4b), but the fluorescence of TTVBA nanospheres was vanishingly weak in this solvent compared with that in $1 \%$ DMSO/toluene (Fig. S16). Therefore, we hypothesized 

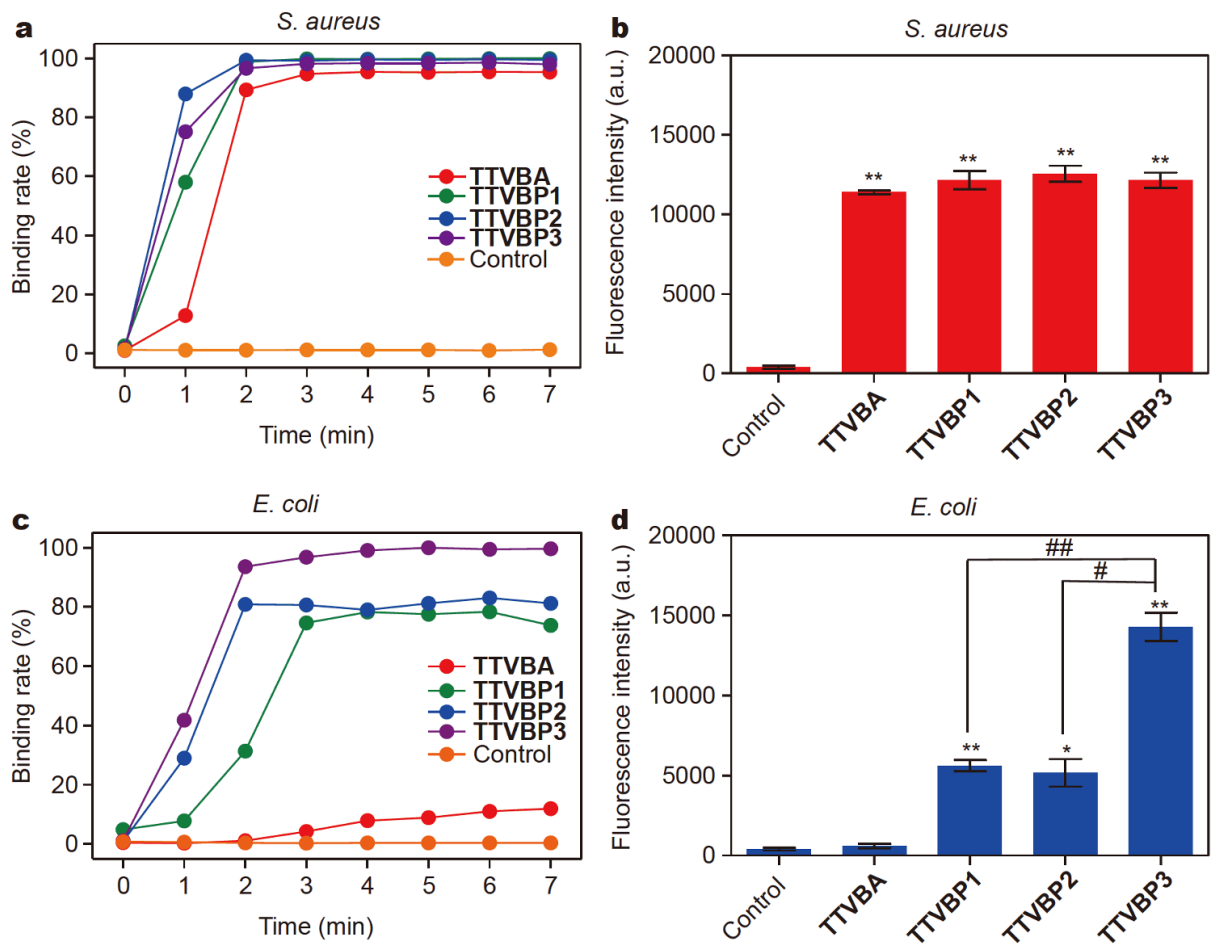

Figure 4 Binding kinetics between AIE-PSs and (a) S. aureus or (c) E. coli, and the fluorescence intensities of (b) S. aureus and (d) E. coli after 5-min incubation with AIE-PSs $\left(10^{-5} \mathrm{~mol} \mathrm{~L}^{-1}\right)$. Data are given as mean $\pm \mathrm{SE}(n=3) .{ }^{\star}$ and ${ }^{\star \star}$ indicate significant differences $(P<0.05$ and $P<0.01$, respectively) from the corresponding control group. \# and \#\# indicate significant differences $(P<0.05$ and $P<0.01$, respectively) between two groups.

that TTVBA nanospheres did not accumulate on the surface of $S$. aureus. Furthermore, the intramolecular motions of AIE-PSs, which will consume the excited state energy, cannot be restricted on the bacterial membrane surface, and thus no fluorescence signal can be detected on the bacterial surface if AIE-PSs only bind to the bacterial surface [45]. Taken together, we assumed that TTVBA nanospheres were decomposed on the hydrophobic surface of $S$. aureus, and the resulting TTVBA molecules intercalated into the porous cell wall, keeping the zeta potential of $S$. aureus unchanged.

In virtue of strong fluorescence of our AIE-PSs on bacteria detected by flow cytometer, the fluorescence imaging of bacteria was viewed under a CLSM after mixing bacteria with AIE-PSs without washing. As shown in Fig. 5a, when S. aureus were mixed with TTVBA, the fluorescence of $S$. aureus can be clearly visualized, while that of E. coli cannot be seen when E. coli were mixed with TTVBA or TTVBA plus $S$. aureus, demonstrating that TTVBA is more selective for $S$. aureus than for $E$. coli. The luminescent intensities of $S$. aureus treated with TTVBP1-3 were similar (Fig. 5b), while the luminescent intensity of $E$. coli treated with TTVBP3 was significantly enhanced compared with that of TTVBP1 or TTVBP2 (Fig. 5c). These phenomena are consistent with the high efficiency of TTVBP1-3 against $S$. aureus and the best killing effect of TTVBP3 on E. coli, demonstrating that the binding ability of AIE-PSs to bacteria affects its fluorescence intensity and photodynamic antibacterial ability. Importantly, all samples were prepared by simple mixing, whereas all images had outstanding image contrast to the background, demonstrating the excellent wash-free imaging ability of TTVBA and TTVBP1-3 toward bacteria.

\section{Stability of AIE-PSs}

The photostability of AIE-PS on $S$. aureus was tested by continuous irradiation $\left(\lambda_{\text {ex }}=488 \mathrm{~nm}\right)$ and sequential scanning with CLSM every $5 \mathrm{~s}$ for 120 times. As shown in Fig. 6a, TTVBP1-3 have higher photobleaching resistances than TTVBA, which is probably due to the reactive carboxylic acid group toward the adjacent vinyl group in TTVBA, or the instability of TTVBA nanospheres under illumination. The relatively weak photostability of TTVBA explained why the fluorescence intensity of TTVBA on $S$. aureus was similar to 

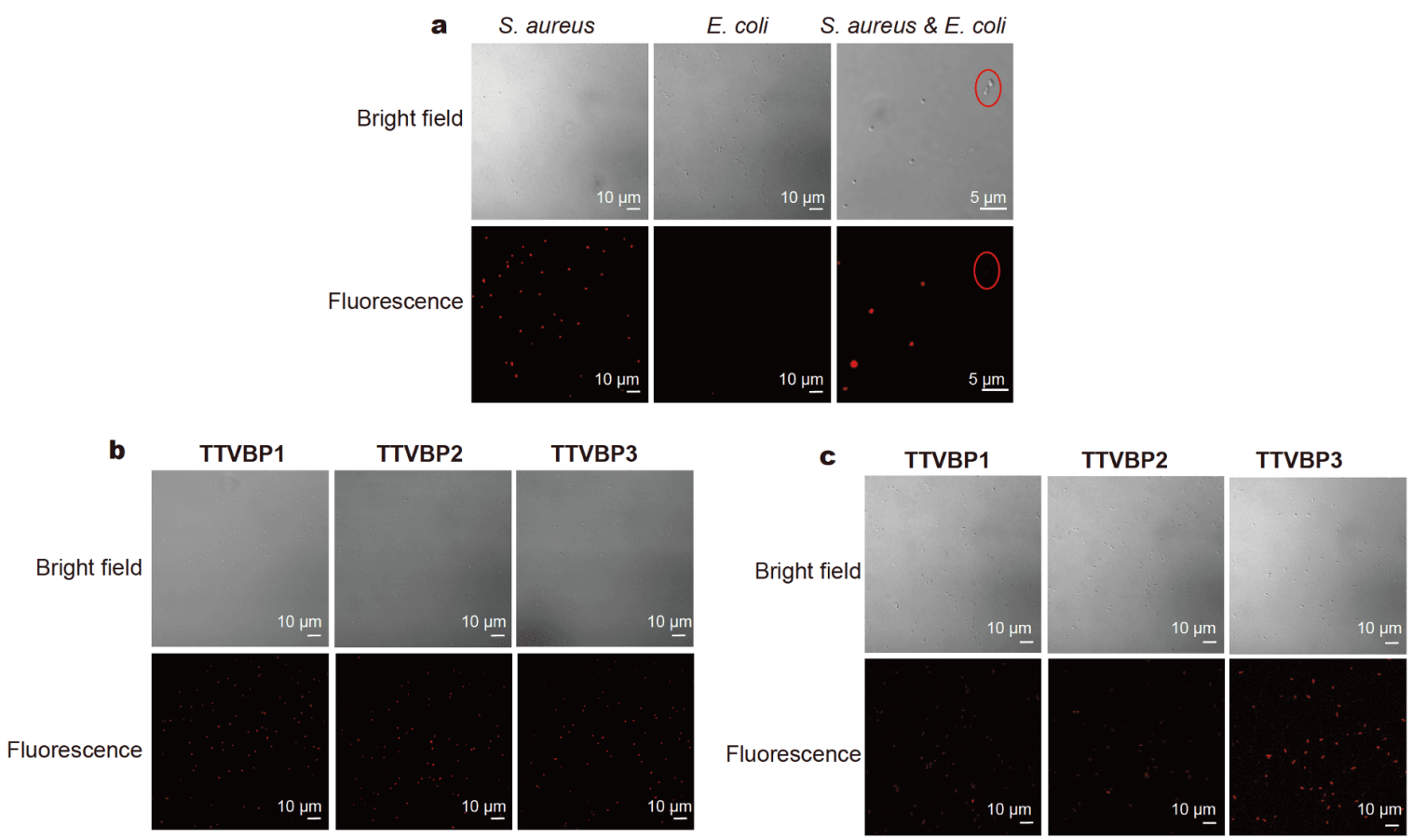

Figure 5 Bacterial imaging after mixing with AIE-PSs. (a) Selective bacterial imaging capability of TTVBA under a CLSM. (b) S. aureus and (c) E. coli imaging after mixing with AIE-PSs without washing.
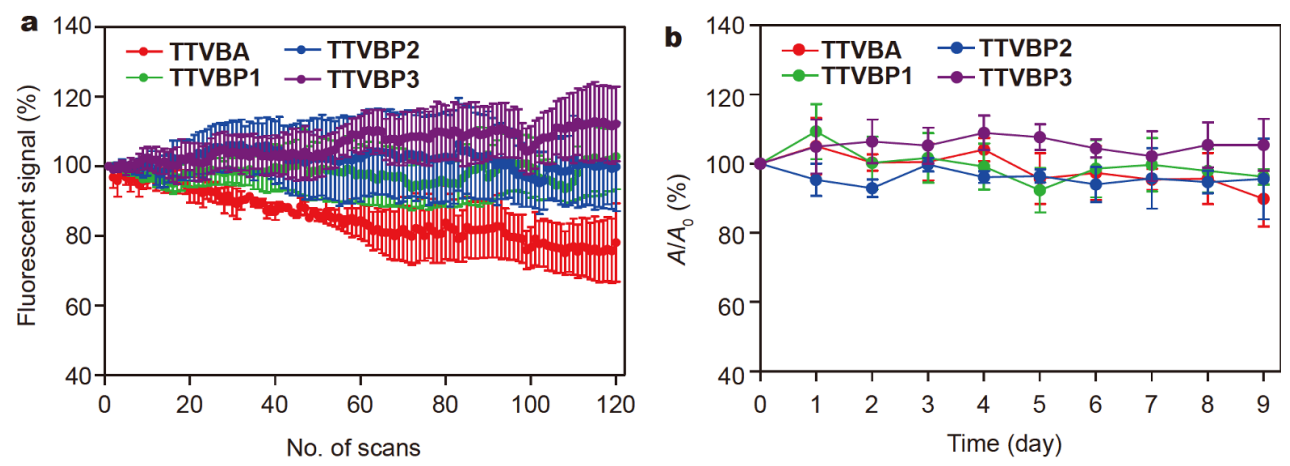

Figure 6 Stability of AIE-PSs investigated $\left(10 \mu \mathrm{mol} \mathrm{L}^{-1}\right)$. (a) Photostability of AIE-PSs on S. aureus. (b) Dark stability of AIE-PSs in PBS. $A_{0}$ and $A$ are the absorption peak values at $450 \mathrm{~nm}$ in PBS on and after the first day, respectively. Data are given as mean \pm SE $(n=3)$.

TTVBP1-3, but its bactericidal effect on $S$. aureus was inferior to TTVBP1-3.

Meanwhile, the dark stability of AIE-PSs in PBS was measured by tracking their daily light absorptions for 9 consecutive days at room temperature. As shown in Fig. $6 \mathrm{~b}$, the intensity of the absorption peak $(450 \mathrm{~nm})$ remained unchanged, indicating that these AIE-PSs were stable in PBS.

\section{Biocompatibility test}

The biocompatibility of above AIE-PS was evaluated by acute toxicity test on human normal hepatocyte cell line
(L-O2). In brief, different concentrations of AIE-PSs were incubated with $\mathrm{L}-\mathrm{O} 2$ cells for $2 \mathrm{~h}$, followed by irradiation with white light $\left(20 \mathrm{~mW} \mathrm{~cm}^{-2}\right)$ for $40 \mathrm{~min}$. As illustrated in Fig. 7, no cytotoxicity or dark toxicity was observed among AIE-PSs investigated at concentrations up to $12.5 \mu \mathrm{mol} \mathrm{L}{ }^{-1}$. TTVBP2 showed slight cytotoxicity at a concentration of $12.5 \mu \mathrm{mol} \mathrm{L}^{-1}$ which is 100 times higher than its effective antibacterial dose $\left(125 \mathrm{nmol} \mathrm{L}^{-1}\right)$. Therefore, these AIE-PSs have excellent biocompatibility.

\section{CONCLUSION}

TTVBA, a nitrobenzoic acid-based AIE-PS with high 

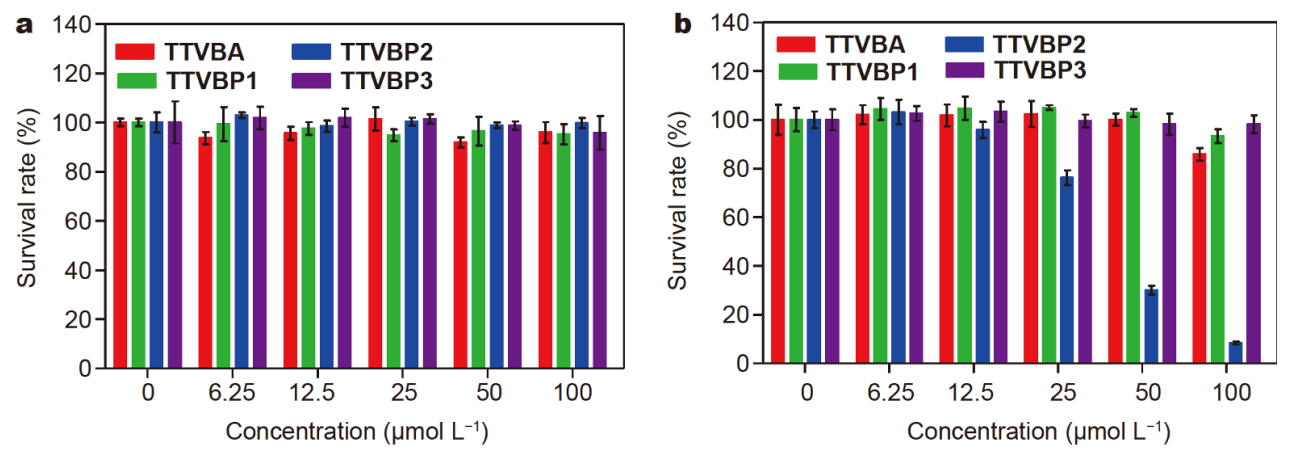

Figure 7 Biocompatibilities of AIE-PSs investigated. (a) Dark toxicity and (b) phototoxicity of AIE-PSs on human normal hepatocyte cell line (L$\mathrm{O} 2)$. Phototoxicity was performed under white-light irradiation $\left(20 \mathrm{~mW} \mathrm{~cm}^{-2}, 40 \mathrm{~min}\right)$. Data are given as mean $\pm \mathrm{SE}(n=3)$.

ROS generation ability, was rationally designed and efficiently prepared without the need of further purification. The negatively charged TTVBA nanospheres selectively imaged and killed sphere-shaped S. aureus, showing their narrow-spectrum antibacterial ability. When coupled with piperazine, TTVBA was easily extended to TTVBP, which was then alkylated to give TTVBP1-3 with positive charges. TTVBP1-3 can efficiently image and kill both $S$. aureus and E. coli. In particular, TTVBP2 killed $3.0 \log _{10}$ of $S$. aureus at a very low concentration $\left(125 \mathrm{nmol} \mathrm{L}^{-1}\right)$, TTVBP3 killed $4.7 \log _{10}$ of $S$. epidermidis at a concentration of $1 \mu \mathrm{mol} \mathrm{L}^{-1}$ and $3.8 \log _{10}$ of E. coli at $5 \mu \mathrm{mol} \mathrm{L}{ }^{-1}$, which makes them among the most effective antibacterial AIE-PSs reported so far. Meanwhile, these AIE-PSs have good photostability and wash-free imaging ability for bacteria with strong contrast to the background. The antibacterial effects of these AIE-PSs were directly proportional to the fluorescence intensity of the bacteria bound with them, suggesting that the bacterial binding ability of AIE-PS determines its photodynamic antibacterial ability. These entire successful examples demonstrate that TTVBA is an extendable AIE-PS backbone with excellent photodynamic and imaging capabilities, and it can be easily extended to AIE-PSs with various functionalities in biomedical studies, including biological imaging, antibacterial and antitumor functions. Moreover, the strong binding of these AIE-PSs to bacteria indicates that alkylated piperazine with positive charges is an effective anchoring group for bacterial membrane, which successfully improves the antibacterial efficacy of AIE-PSs.

Received 1 January 2021; accepted 22 February 2021; published online 28 May 2021

1 Blair JMA, Webber MA, Baylay AJ, et al. Molecular mechanisms of antibiotic resistance. Nat Rev Microbiol, 2015, 13: 42-51
2 Moore CE. Changes in antibiotic resistance in animals. Science, 2019, 365: 1251-1252

3 Yang B, Gao F, Li Z, et al. Selective entropy gain-driven adsorption of nanospheres onto spherical bacteria endows photodynamic treatment with narrow-spectrum activity. J Phys Chem Lett, 2020, 11: $2788-2796$

4 Shi X, Sung SHP, Chau JHC, et al. Killing $\mathrm{G}(+)$ or $\mathrm{G}(-)$ bacteria? The important role of molecular charge in AIE-active photosensitizers. Small Methods, 2020, 4: 2000046

5 Dai T, Ye F, Hu P, et al. A strategy for enhanced tumor targeting of photodynamic therapy based on Escherichia coli-driven drug delivery system. Sci China Mater, 2021, 64: 232-240

6 Jia HR, Zhu YX, Chen Z, et al. Cholesterol-assisted bacterial cell surface engineering for photodynamic inactivation of Gram-positive and Gram-negative bacteria. ACS Appl Mater Interfaces, 2017, 9: 15943-15951

7 Li J, Sun W, Yang Z, et al. Rational design of self-assembled cationic porphyrin-based nanoparticles for efficient photodynamic inactivation of bacteria. ACS Appl Mater Interfaces, 2020, 12: 54378-54386

8 Sun YD, Zhu YX, Zhang X, et al. Role of cholesterol conjugation in the antibacterial photodynamic therapy of branched polyethylenimine-containing nanoagents. Langmuir, 2019, 35: 1432414331

9 Guan Q, Fu DD, Li YA, et al. Bodipy-decorated nanoscale covalent organic frameworks for photodynamic therapy. iScience, 2019, 14: 180-198

10 Hong Y, Lam JW, Tang BZ. Aggregation-induced emission. Chem Soc Rev, 2011, 40: 5361-538

11 Yang W, Ling B, Hu B, et al. Synergistic N-heterocyclic carbene/ palladium-catalyzed umpolung 1,4-addition of aryl iodides to enals. Angew Chem Int Ed, 2020, 59: 161-166

$12 \mathrm{Xu} \mathrm{W}$, Lee MMS, Nie JJ, et al. Three-pronged attack by homologous far-red/NIR AIEgens to achieve $1+1+1>3$ synergistic enhanced photodynamic therapy. Angew Chem Int Ed, 2020, 59: 9610-9616

13 Li X, Lee S, Yoon J. Supramolecular photosensitizers rejuvenate photodynamic therapy. Chem Soc Rev, 2018, 47: 1174-1188

14 Li S, Zou Q, Li Y, et al. Smart peptide-based supramolecular photodynamic metallo-nanodrugs designed by multicomponent coordination self-assembly. J Am Chem Soc, 2018, 140: 1079410802

15 Mei J, Leung NLC, Kwok RTK, et al. Aggregation-induced emis- 
sion: Together we shine, united we soar! Chem Rev, 2015, 115: $11718-11940$

16 Jiang N, Shen T, Sun JZ, et al. Aggregation-induced emission: Right there shining. Sci China Mater, 2019, 62: 1227-1235

17 Zhao L, Liu Y, Xing R, et al. Supramolecular photothermal effects: A promising mechanism for efficient thermal conversion. Angew Chem Int Ed, 2019, 59: 3793-3801

18 Luo J, Xie Z, Lam JWY, et al. Aggregation-induced emission of 1methyl-1,2,3,4,5-pentaphenylsilole. Chem Commun, 2001, 21: 1740-1741

19 Qi J, Duan X, Liu W, et al. Dragonfly-shaped near-infrared AIEgen with optimal fluorescence brightness for precise image-guided cancer surgery. Biomaterials, 2020, 248: 120036

20 Chong KC, Hu F, Liu B. AIEgen bioconjugates for specific detection of disease-related protein biomarkers. Mater Chem Front, 2019, 3: 12-24

21 Cai X, Liu B. Aggregation-induced emission: Recent advances in materials and biomedical applications. Angew Chem Int Ed, 2020, 59: 9868-9886

22 Liu Z, Zou H, Zhao Z, et al. Tuning organelle specificity and photodynamic therapy efficiency by molecular function design. ACS Nano, 2019, 13: 11283-11293

23 Wang D, Lee MMS, Shan G, et al. Highly efficient photosensitizers with far-red/near-infrared aggregation-induced emission for in vitro and in vivo cancer theranostics. Adv Mater, 2018, 30: 1802105

24 Han W, Zhang S, Deng R, et al. Self-assembled nanostructured photosensitizer with aggregation-induced emission for enhanced photodynamic anticancer therapy. Sci China Mater, 2019, 63: 136146

25 Xu M, Wang X, Wang Q, et al. Analyte-responsive fluorescent probes with AIE characteristic based on the change of covalent bond. Sci China Mater, 2019, 62: 1236-1250

26 Liu S, Li Y, Kwok RTK, et al. Structural and process controls of AIEgens for NIR-II theranostics. Chem Sci, 2021, 12: 3427-3436

27 Chen J, Gao M, Wang L, et al. Aggregation-induced emission probe for study of the bactericidal mechanism of antimicrobial peptides. ACS Appl Mater Interfaces, 2018, 10: 11436-11442

28 Roy E, Nagar A, Chaudhary S, et al. AIEgen-based fluorescent nanomaterials for bacterial detection and its inhibition. ChemistrySelect, 2020, 5: 722-735

29 Zhao E, Chen Y, Chen S, et al. A luminogen with aggregationinduced emission characteristics for wash-free bacterial imaging, high-throughput antibiotics screening and bacterial susceptibility evaluation. Adv Mater, 2015, 27: 4931-4937

30 Zhao N, Li P, Zhuang J, et al. Aggregation-induced emission luminogens with the capability of wide color tuning, mitochondrial and bacterial imaging, and photodynamic anticancer and antibacterial therapy. ACS Appl Mater Interfaces, 2019, 11: 1122711237

31 Zehra N, Dutta D, Malik AH, et al. Fluorescence resonance energy transfer-based wash-free bacterial imaging and antibacterial application using a cationic conjugated polyelectrolyte. ACS Appl Mater Interfaces, 2018, 10: 27603-27611

32 Zhu S, Wang X, Yang Y, et al. Conjugated polymer with aggregation-directed intramolecular Förster resonance energy transfer enabling efficient discrimination and killing of microbial pathogens. Chem Mater, 2018, 30: 3244-3253

33 Panigrahi A, Are VN, Jain S, et al. Cationic organic nanoaggregates as AIE luminogens for wash-free imaging of bacteria and broadspectrum antimicrobial application. ACS Appl Mater Interfaces,
2020, 12: 5389-5402

34 Lee MMS, Xu W, Zheng L, et al. Ultrafast discrimination of Grampositive bacteria and highly efficient photodynamic antibacterial therapy using near-infrared photosensitizer with aggregation-induced emission characteristics. Biomaterials, 2020, 230: 119582

35 He X, Yang Y, Guo Y, et al. Phage-guided targeting, discriminative imaging, and synergistic killing of bacteria by AIE bioconjugates. J Am Chem Soc, 2020, 142: 3959-3969

36 Li J, Wang J, Li H, et al. Supramolecular materials based on AIE luminogens (AIEgens): Construction and applications. Chem Soc Rev, 2020, 49: 1144-1172

37 Zhang Y, Zhao X, Li Y, et al. A fluorescent photosensitizer with far $\mathrm{red} /$ near-infrared aggregation-induced emission for imaging and photodynamic killing of bacteria. Dyes Pigments, 2019, 165: 53-57

38 Chen X, Huang L, Jia Y, et al. AIE-based theranostic probe for sequential imaging and killing of bacteria and cancer cells. Adv Opt Mater, 2020, 8: 1902191

39 Chen $\mathrm{H}$, Li S, Wu M, et al. Membrane-anchoring photosensitizer with aggregation-induced emission characteristics for combating multidrug-resistant bacteria. Angew Chem Int Ed, 2020, 59: 632636

40 Merchat M, Bertolini G, Giacomini P, et al. Meso-substituted cationic porphyrins as efficient photosensitizers of Gram-positive and Gram-negative bacteria. J Photochem Photobiol B-Biol, 1996, 32: $153-157$

41 Zhang Y, Zheng K, Chen Z, et al. Rapid killing of bacteria by a new type of photosensitizer. Appl Microbiol Biotechnol, 2017, 101: 4691-4700

42 Brown ED, Wright GD. Antibacterial drug discovery in the resistance era. Nature, 2016, 529: 336-343

43 Perros M. A sustainable model for antibiotics. Science, 2015, 347: 1062-1064

44 Feng G, Yuan Y, Fang H, et al. A light-up probe with aggregationinduced emission characteristics (AIE) for selective imaging, naked-eye detection and photodynamic killing of Gram-positive bacteria. Chem Commun, 2015, 51: 12490-12493

45 Kang M, Zhou C, Wu S, et al. Evaluation of structure-function relationships of aggregation-induced emission luminogens for simultaneous dual applications of specific discrimination and efficient photodynamic killing of Gram-positive bacteria. J Am Chem Soc, 2019, 141: 16781-16789

46 Bennett LE, Ghiggino KP, Henderson RW. Singlet oxygen formation in monomeric and aggregated porphyrin c. J Photochem Photobiol B-Biol, 1989, 3: 81-89

47 Yan D, Wu Q, Wang D, et al. Innovative synthetic procedures for luminogens showing aggregation-induced emission. Angew Chem Int Ed, 2021, doi: 10.1002/anie.202006191

48 Liu GJ, Tian SN, Li CY, et al. Aggregation-induced-emission materials with different electric charges as an artificial tongue: Design, construction, and assembly with various pathogenic bacteria for effective bacterial imaging and discrimination. ACS Appl Mater Interfaces, 2017, 9: 28331-28338

49 Li Y, Hu X, Tian S, et al. Polyion complex micellar nanoparticles for integrated fluorometric detection and bacteria inhibition in aqueous media. Biomaterials, 2014, 35: 1618-1626

50 Li Q, Wu Y, Lu H, et al. Construction of supramolecular nanoassembly for responsive bacterial elimination and effective bacterial detection. ACS Appl Mater Interfaces, 2017, 9: 1018010189

51 Ward JS, Nobuyasu RS, Batsanov AS, et al. The interplay of 
thermally activated delayed fluorescence (TADF) and room temperature organic phosphorescence in sterically-constrained donoracceptor charge-transfer molecules. Chem Commun, 2016, 52: 2612-2615

52 Grabowski ZR, Rotkiewicz K, Rettig W. Structural changes accompanying intramolecular electron transfer: Focus on twisted intramolecular charge-transfer states and structures. Chem Rev, 2003, 103: 3899-4032

53 Aoki S, Kagata D, Shiro M, et al. Metal chelation-controlled twisted intramolecular charge transfer and its application to fluorescent sensing of metal ions and anions. J Am Chem Soc, 2004, 126: 13377-13390

54 Zhang Q, Kuwabara H, Potscavage Jr. WJ, et al. Anthraquinonebased intramolecular charge-transfer compounds: Computational molecular design, thermally activated delayed fluorescence, and highly efficient red electroluminescence. J Am Chem Soc, 2014, 136: $18070-18081$

55 DeRosa M. Photosensitized singlet oxygen and its applications. Coord Chem Rev, 2002, 233-234: 351-371

56 Devasagayam TPA, Kamat JP. Biological significance of singlet oxygen. Indian J Exp Biol, 2002, 40: 680-692

57 Lan M, Zhao S, Liu W, et al. Photosensitizers for photodynamic therapy. Adv Healthcare Mater, 2019, 8: 1900132

58 George S, Hamblin MR, Kishen A. Uptake pathways of anionic and cationic photosensitizers into bacteria. Photochem Photobiol Sci, 2009, 8: 788-795

59 Minnock A, Vernon DI, Schofield J, et al. Mechanism of uptake of a cationic water-soluble pyridinium zinc phthalocyanine across the outer membrane of Escherichia coli. Antimicrob Agents Chemother, 2000, 44: 522-527

60 Yuan H, Liu Z, Liu L, et al. Cationic conjugated polymers for discrimination of microbial pathogens. Adv Mater, 2014, 26: 43334338

61 Bai H, Yuan H, Nie C, et al. A supramolecular antibiotic switch for antibacterial regulation. Angew Chem Int Ed, 2015, 54: 1320813213

Acknowledgements This work was supported by the National Natural Science Foundation of China (81572944 and 81971983), the CAS/ SAFEA International Partnership Program for Creative Research Teams, the High-Level Entrepreneurship and Innovation Talents Projects in Fujian Province (2018-8-1), and the FJIRSM\&IUE Joint Research Fund (RHZX-2018-004).

Author contributions Wang H, Pan X and Chen $\mathrm{Z}$ conceived and designed the project; Pan X designed the AIE PSs; Wang $\mathrm{H}$ and Wang Y synthesized the AIE PSs; Wang $\mathrm{H}$ performed the experiments; Wang $\mathrm{H}$ and Pan X analyzed the data; Liu W, Dai T and Yuan B provided the technical support; Pan X, Chen Z, Wang H and Chen X finished the writing.

Conflict of interest The authors declare that they have no conflict of interest.

Supplementary information online version of the paper.

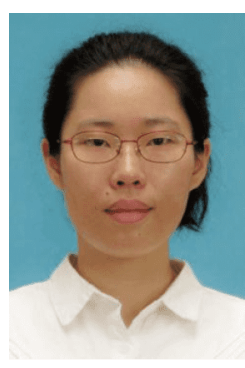

Huanhuan Wang received her BSc degree in chemistry from Shanxi University in 2017. She is currently a postgraduate under the supervision of Prof. Zhuo Chen at Fujian Institute of Research on the Structure of Matter (FJIRSM), Chinese Academy of Sciences (CAS). Her current research focuses on the design and synthesis of aggregation-induced emission photosensitizers and their antibacterial applications.

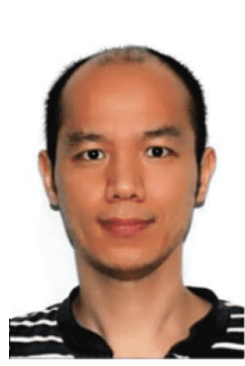

Xiaohong Pan received his $\mathrm{PhD}$ degree from the University of Pittsburgh in 2011 and his BSc degree from the University of Science and Technology of China in 2002. From 2011 to 2018, he worked at the Scripps Research in Florida as a research associate. He is currently an associate professor at FJIRSM, CAS. His research interest focuses on the design and synthesis of new photosensitizers for photodynamic therapy.

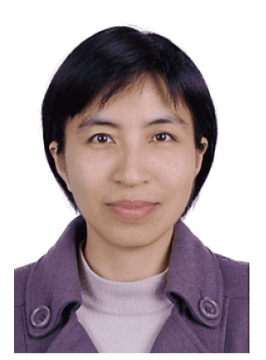

Zhuo Chen received her $\mathrm{PhD}$ degree from FJIRSM, CAS, following her MD degree from Fujian Medical University and her BSc degree from Chinese Pharmaceutical University. From 2001 to 2008, she worked on molecular pharmacology \& therapeutics in Loyola University Chicago as a research associate. Her research focuses on tumor detection and targeted photodynamic therapy, including design and synthesis of new anticancer drug entities and evaluation of their pharmacological effects through experiments in cells and animals.

\section{基于硝基苯甲酸结构的新型AIE光敏剂的光动力 抗菌作用}

王欢欢 ${ }^{1,2}$, 潘小宏 ${ }^{1 *}$, 王亚琪 ${ }^{1}$, 刘雯珍 ${ }^{1}$, 戴涛 ${ }^{1,2}$, 袁彬彬 ${ }^{1}$, 陈学元 ${ }^{1,2}$, 陈卓 12

摘要 光动力疗法在抗菌领域中的应用备受关注. 传统的光敏剂在 水性介质中容易聚集, 从而减少活性氧的产生并严重影响其光动 力抗菌疗效. 为了减少传统光敏剂的聚集, 研究人员做出了许多努 力. 与之相反, 聚集诱导发光型的光敏剂(AIE-PSs)利用其聚集的优 势, 不仅增加了活性氧产量, 而且增强了荧光强度. 然而目前有关抗 菌型AIE-PSs的研究仍处在发展阶段, 相关报道也非常有限. 我们在 此首次报道了系列基于硝基苯甲酸结构的高效抗菌型AIE-PSs. 其 中带负电荷的TTVBA不仅可选择性地灭杀球形细菌(如金黄色葡 萄球菌), 而且易于被扩展成多种带正电荷且具有广谱抗菌性能的 AIE-PSs(如TTVBP1-3). 我们发现, TTVBP2在 $125 \mathrm{nmol} \mathrm{L}^{-1}$ 的低 浓度下，即可灭杀3.0 $\log _{10}$ 金黄色葡萄球菌; TTVBP3 在 $1 \mu \mathrm{mol} \mathrm{L} \mathrm{L}^{-1}$ 浓度下可灭杀 $4.7 \quad \log _{10}$ 表皮葡萄球菌, 在 $5 \mu \mathrm{mol} \mathrm{L}{ }^{-1}$ 浓度下可灭杀 $3.8 \log _{10}$ 大肠杆菌, 成为目前已报道的最有效的抗菌AIE-PSs. 此外, 这些AIE-PSs直接与细菌混合后, 即对细菌具有出色的免洗成像能 力. 因此, 我们认为基于硝基苯甲酸结构的 TTVBA为未来高效抗 菌光敏剂的设计提供了崭新思路. 